\title{
Immediate Implant Placement by Dual Stabilization Technique
}

\author{
${ }^{1}$ Charles D Schlesinger, ${ }^{2}$ Rajiv Saini
}

\begin{abstract}
The dental implant provides a realistic treatment alternative for rehabilitation of patients with lost teeth. Immediate implant placement following tooth extraction in appropriately selected cases has been considered the optimal procedure option in modern implant dentistry. This articles illustrates a case of immediate implantation in the right maxillary first molar region. The OCO Biomedical dental implant used in this case report is designed for immediate loading using Dual Stabilization ${ }^{\mathrm{TM}}$, an industry unique design feature that biomechanically secures the tip and the collar of the implant into bone. This case report illustrates that it is possible to provide the patient with efficient tooth replacement with a quality immediate dental implant.
\end{abstract}

Keywords: Immediate implants, Dual stabilization, Osseous fixation, OCO biomedical.

How to cite this article: Schlesinger CD, Saini R. Immediate Implant Placement by Dual Stabilization Technique. Int J Experiment Dent Sci 2015;4(1):72-76.

\section{Source of support: Nil}

Conflict of interest: None

\section{INTRODUCTION}

The goal of modern dentistry is to prevent tooth loss and to provide a healthy dentition with optimal functional efficiency, structural balance and esthetic harmony. The dental implant provides a realistic treatment alternative for the rehabilitation of patients with lost teeth. ${ }^{1}$ According to traditional protocols, a 3 to 4 months healing period is required for the consolidation and healing of the extraction socket. Taking into account the prosthetic treatment, patients frequently are required to wait up to 1 year for replacement of a lost tooth. ${ }^{2}$ Planning implant treatment for a hopeless tooth starts prior to extraction. The timing and technique of the extraction, to the timing of implant placement, all play critical roles in treatment

\footnotetext{
${ }^{1}$ Chief Operating Officer and Director, ${ }^{2}$ Associate Professor

${ }^{1}$ Department of Clinical Affairs, OCO Biomedical, Albuquerque New Mexico, USA

${ }^{2}$ Department of Periodontology, Pravara Institute of Medical Sciences, Ahmednagar, Maharashtra, India

Corresponding Author: Charles D Schlesinger, Chief Operating Officer and Director, Department of Clinical Affairs oCO Biomedical, Albuquerque, New Mexico, USA, e-mail: chuck@ocobiomedical.com
}

outcome. The timing of implant placement is the fourth dimension in treatment planning for implants after the three dimensions of available bone. ${ }^{3}$

One such treatment protocol is the placement of implants into fresh extraction sockets, otherwise commonly known as immediate implant placement. Immediate implant placement may be defined as implant placement immediately following tooth extraction and as part of the same surgical procedure, ${ }^{4}$ or as implant placement immediately following extraction of a tooth which must be combined, in most cases, with bone-grafting of the peri-implant socket defects. ${ }^{5}$

Immediate implant placement following tooth extraction in appropriately selected cases has been considered the optimal procedure for the following reasons: the natural healing process are mobilized to the maximum, no bone resorption has taken place yet, drilling is reduced, a number of surgical stages are eliminated, design and construction of prosthesis is simplified, and positive psychological effect on the patient. ${ }^{6,7}$ Here, we report a case of immediate implantation in the right maxillary first molar region and discuss the various factors that predicate success.

\section{CASE REPORT}

A 54 years old male patient with fractured maxillary right first molar visited the OCO Biomedical Clinic, Albuquerque, New Mexico. The crown was temporarily cemented and the whole coronal portion of the tooth was fractured off. Clinical and radiographic examination revealed a fractured tooth with unfavorable prognosis and, therefore, scheduled for extraction. The patient was given a detailed explanation concerning the present state, alternate treatment plans and proposed procedures which included immediate implant placement and early loading. The patient was keen for earliest possible restoration for his tooth and gave the consent for the proposed procedure. Standard clinical assessment was carried out and the patient did not present with any significant medical or systemic issues that would compromise dental implant success. Clinical assessment included an extraoral examination followed by an intraoral evaluation. Presurgical radiographic evaluation was carried out with IOPA for appropriate treatment planning (Fig. 1). The implant used 
in this case was a $6 \times 10$ macro implant (OCO Biomedical Company, Albuquerque, New Mexico, USA).

The surgical procedure was carried out under local anesthesia in sterile surgical conditions (Fig. 2). The tooth was extracted with minimal trauma and was sectioned to facilitate an atraumatic extraction (Figs 3 and 4). After the tooth was extracted, the socket was thoroughly curettaged and cleansed with copious amounts of saline irrigation to remove remnants of periodontal tissue and debris (Fig. 5). The surgeon ensured that there was fresh bleeding from the socket walls before proceeding with the implant site preparations. The site preparations were then carried out according to the implant system protocol.

The drilling procedure was carried out with copious amounts of irrigation and the osteotomy was made apical to the socket depth so as to allow engagement of the apical bone for primary stability. After the pilot hole was drilled into interseptal bone, a paralleling pin was placed to verify position for the final restoration (Fig. 6) and verification of interocclusal space (Figs 7 and 8). Allograft material was grafted to the socket prior to

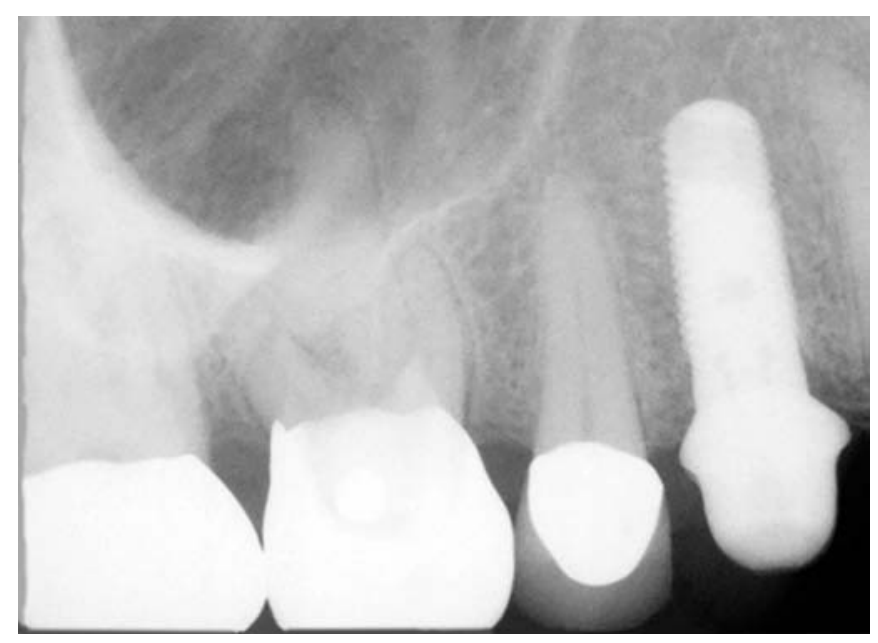

Fig. 1: Preoperative radiograph

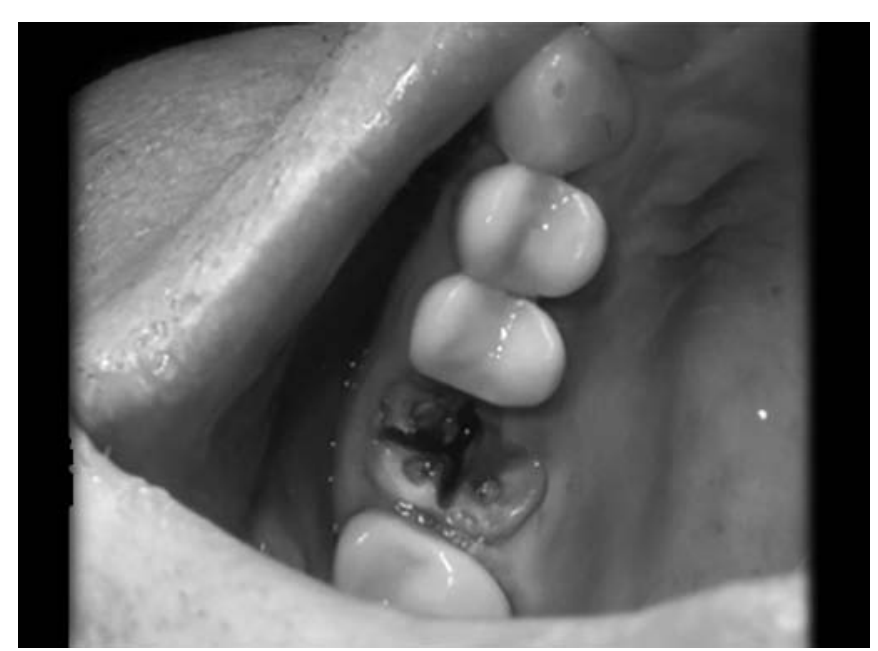

Fig. 3: Tooth sectioned to facilitate an atraumatic extraction implant placement as peri-implant socket graft material (Figs 9 and 10). The $6 \times 10$ macro implant was inserted first with a handpiece at the recommended torque and final seating done manually with a hand wrench (Figs 11 and 12). The surgical site was then secured over the socket/implant by sutures (Fig. 13). Immediate postoperative radiographs were done as a baseline record and to confirm final placement (Fig. 14).

The patient was discharged with postoperative instructions and medications. Follow-up reviews were conducted at 1 week post-surgery (Fig. 15), thereafter, at a month and then removal of cover screw at 2.5 months (Fig. 16). Porcelain fused to gold (PFG) crown was chosen as the final restoration (Figs 17 and 18), and a final seat radiograph was taken (Fig. 19).

\section{DISCUSSION}

The term immediate implant placement refers to the placement of dental implants at the time of tooth removal. Interest in immediate implant placement following tooth extraction has rapidly grown since the first publication on

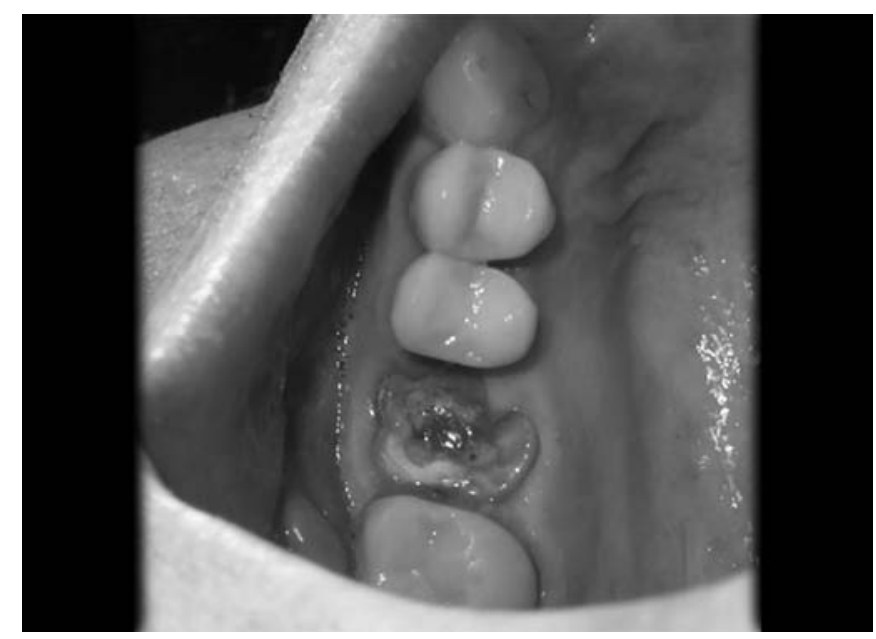

Fig. 2: Preoperative intraoral after removal of coronal portion of the tooth

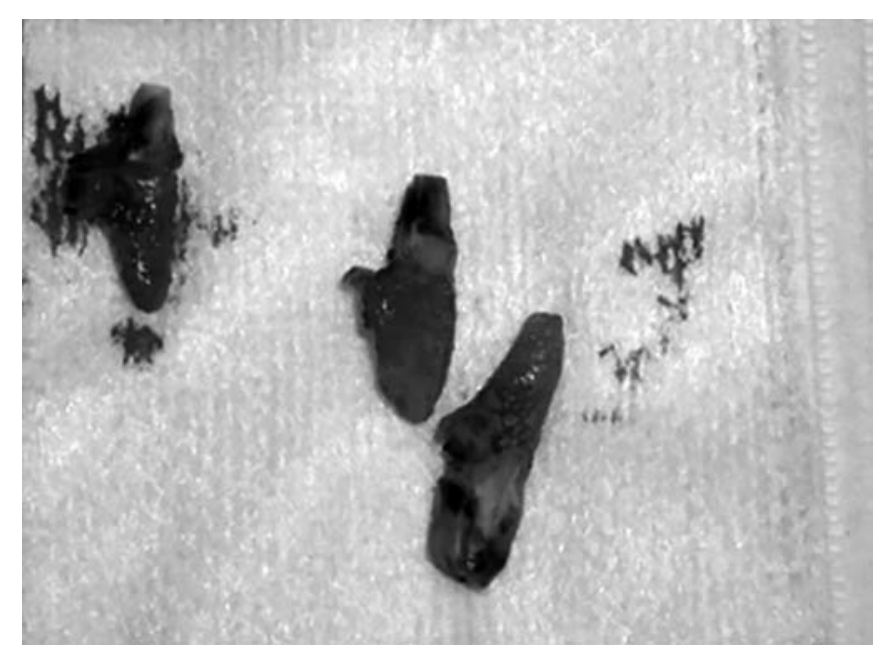

Fig. 4: Sectioned roots after extraction 


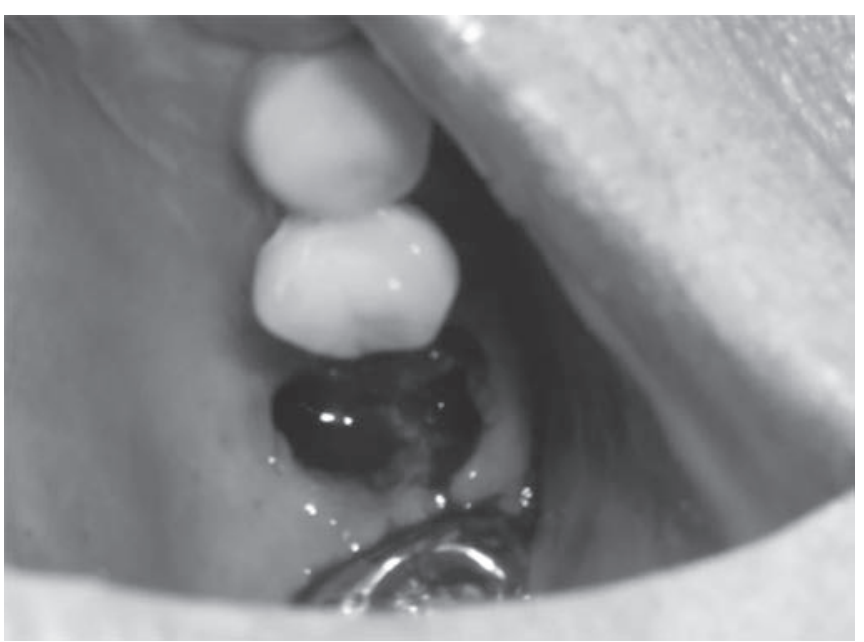

Fig. 5: Extraction site

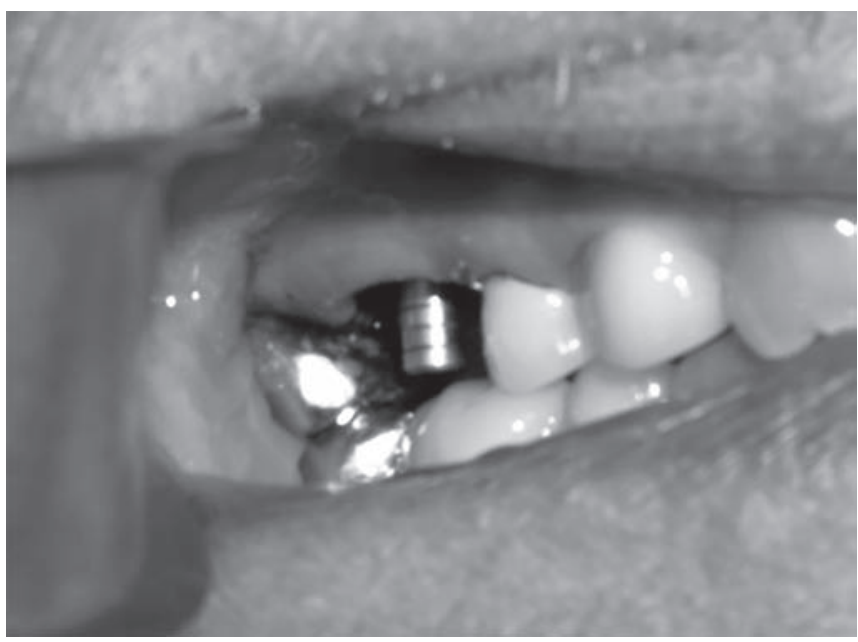

Fig. 7: Verification of interocclusal space with paralleling pin

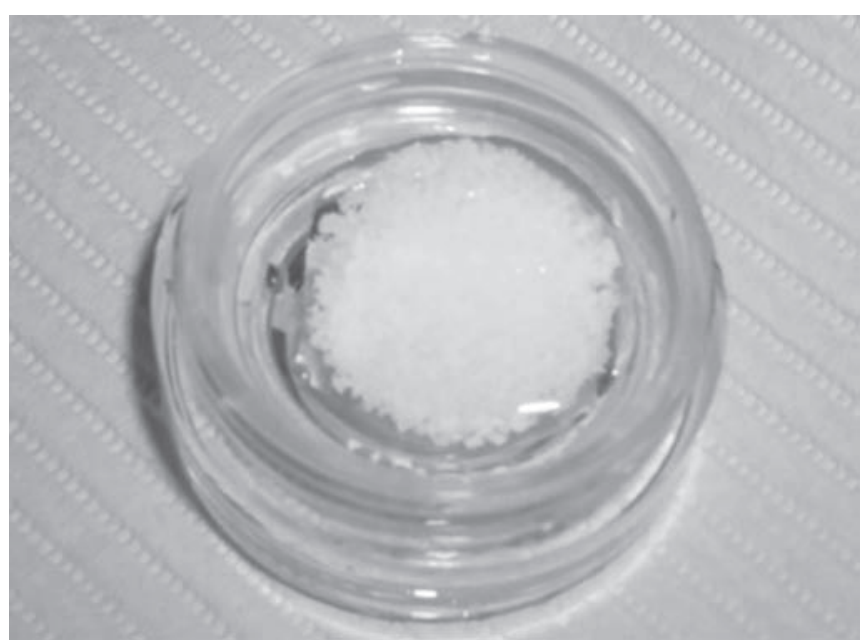

Fig. 9: Xenograft material to graft the socket prior to implant placement

this topic 30 years ago. Immediate implantation is now considered a clinically predictable procedure. ${ }^{8}$ Immediate placement benefits include reduction in surgical procedures, preserving esthetics, conserving bone height and

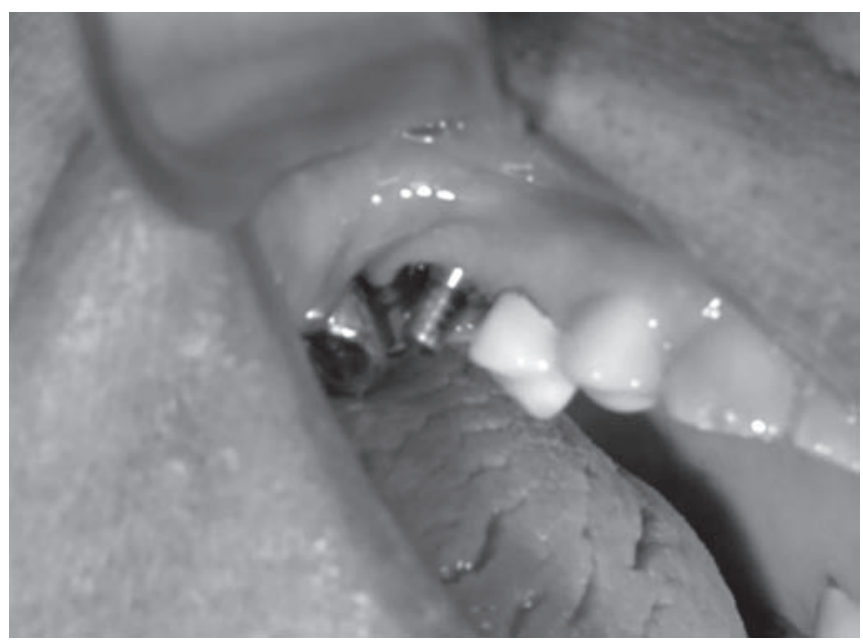

Fig. 6: After pilot hole was drilled in interseptal bone, a paralleling pin was placed to verify position for the final restoration

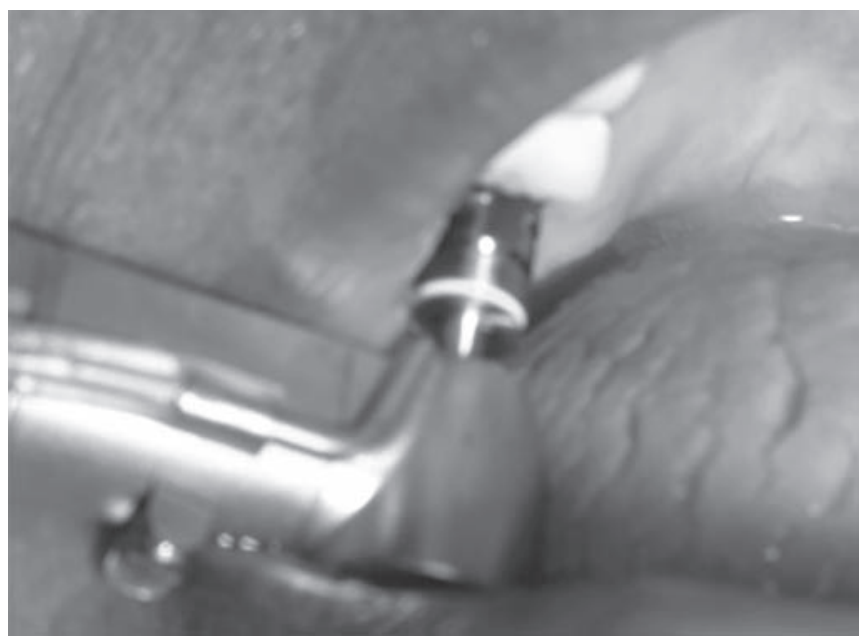

Fig. 8: Final $5.7 \mathrm{~mm}$ osteotomy former

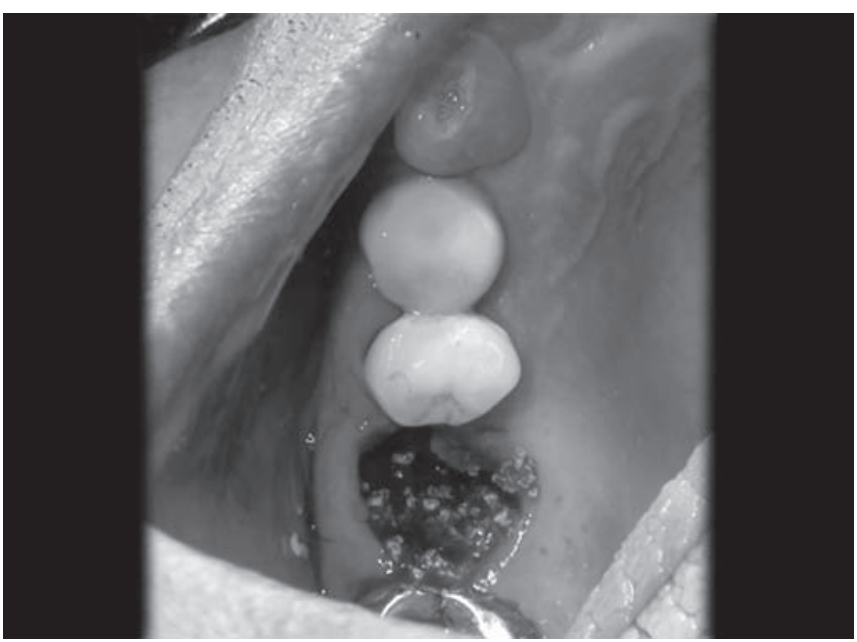

Fig. 10: Grafted site

width and improving patient comfort, acceptance and satisfaction. Furthermore, the healing capability of the fresh extraction site and implant surface characteristics provide improved opportunity for osseointegration. ${ }^{9}$ 


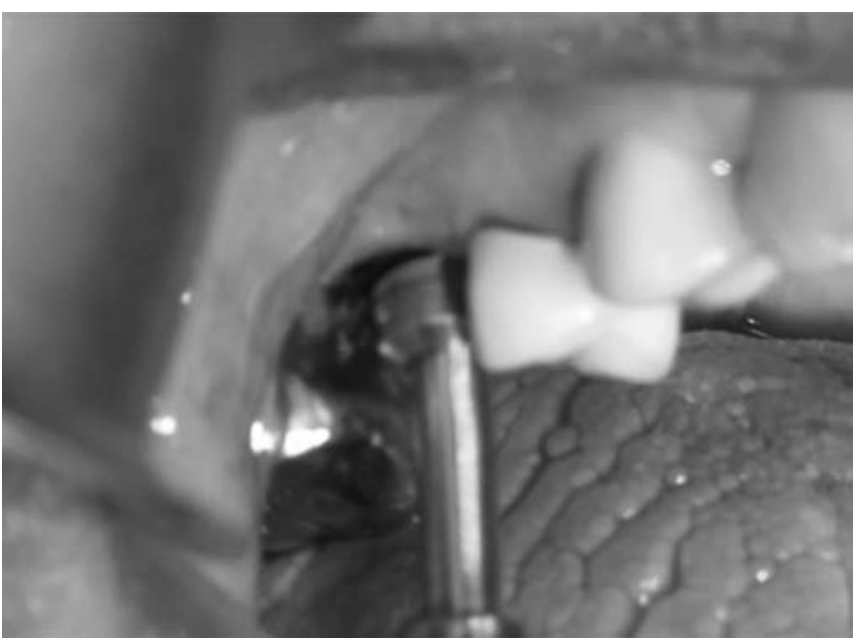

Fig. 11: Insertion of macro implant

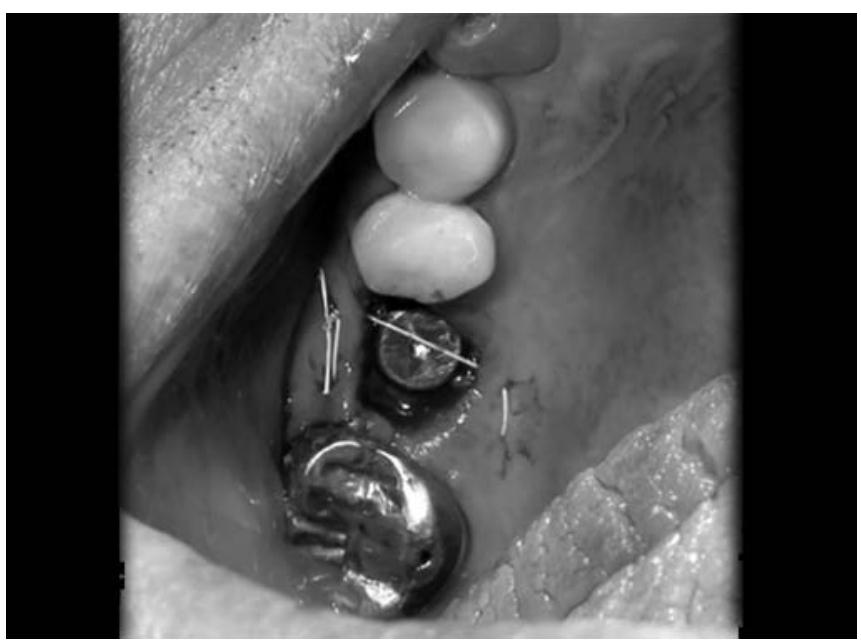

Fig. 13: Final closure of site

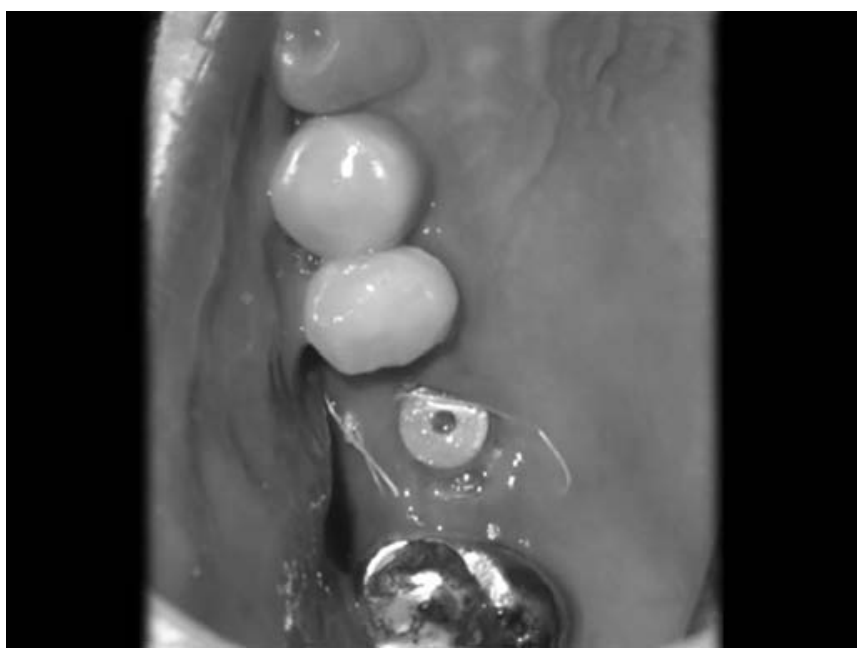

Fig. 15: One week postoperative view

A main factor determining the success of immediate placement is the initial stability of the implant. The extraction site must be evaluated to see whether it is suitable for immediate implant placement. Micro movement between the implant and the surrounding bone should be avoided to allow successful healing to occur. To be

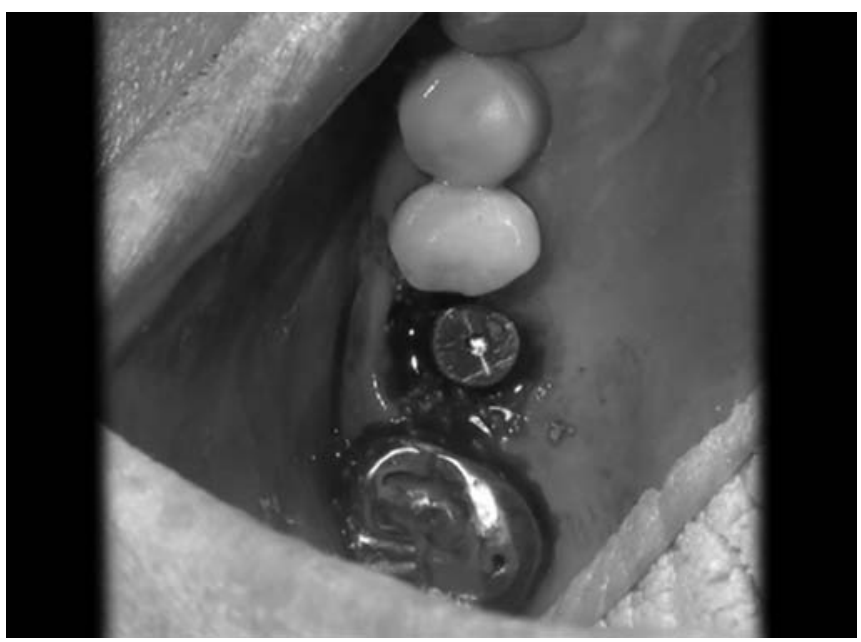

Fig. 12: Implant in place with cover screw

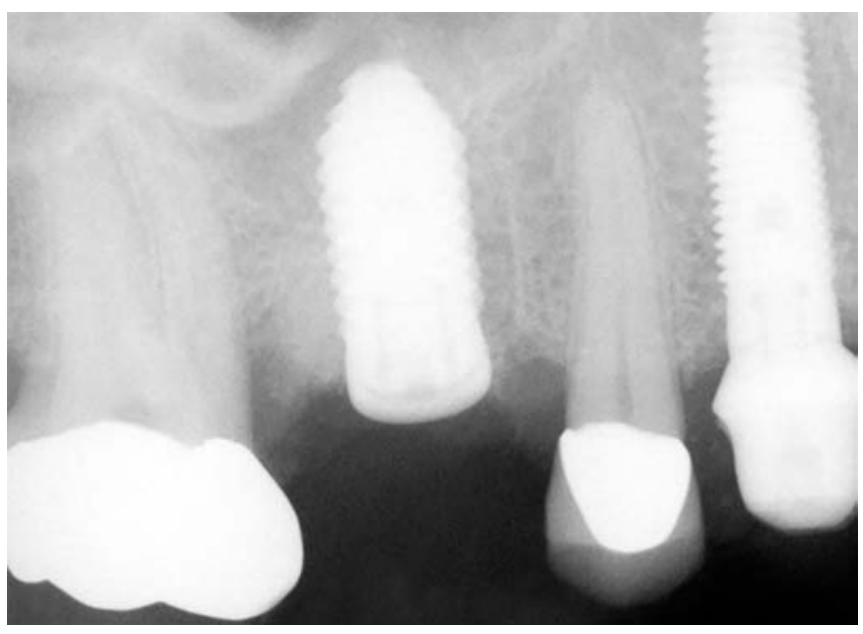

Fig. 14: Radiograph of final placement

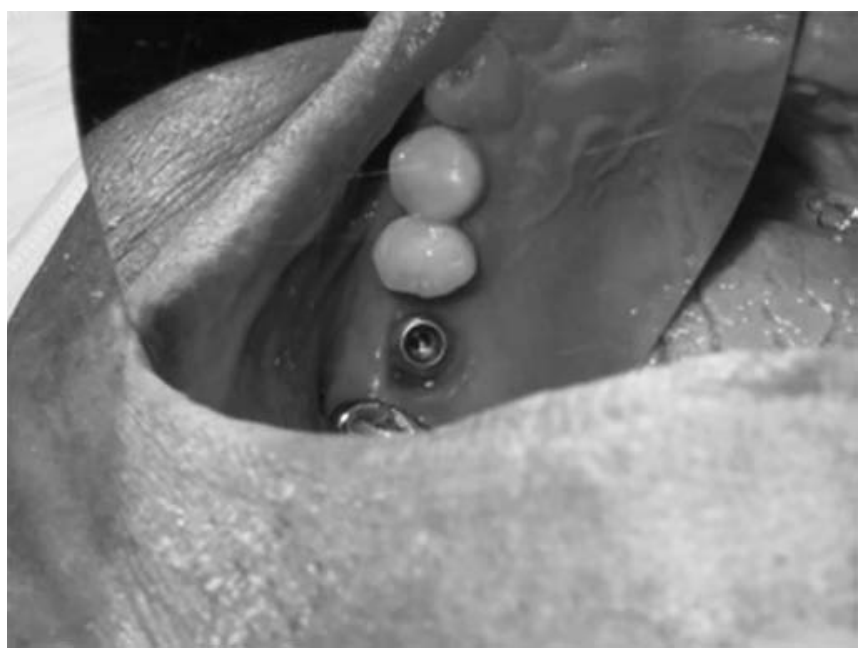

Fig. 16: Removal of cover screw at 2.5 months

successful, implant placement should only be attempted if there is complete resolution of local infection, and if there is enough bone for placement of an appropriately sized implant, in the ideal restorative position and with primary implant stability. Implant placement can be adversely affected by infection in the implantation site, 


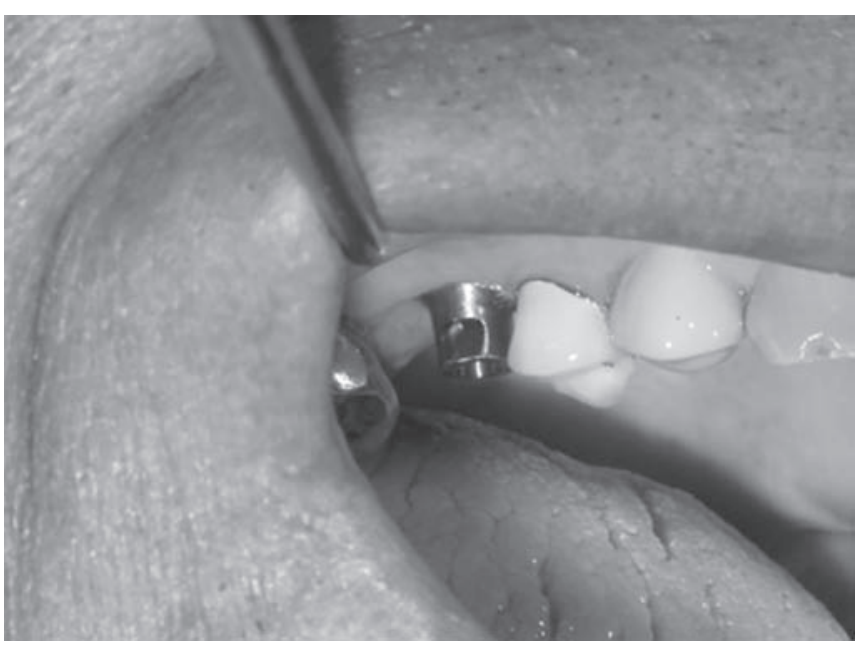

Fig. 17: Placement of final abutment

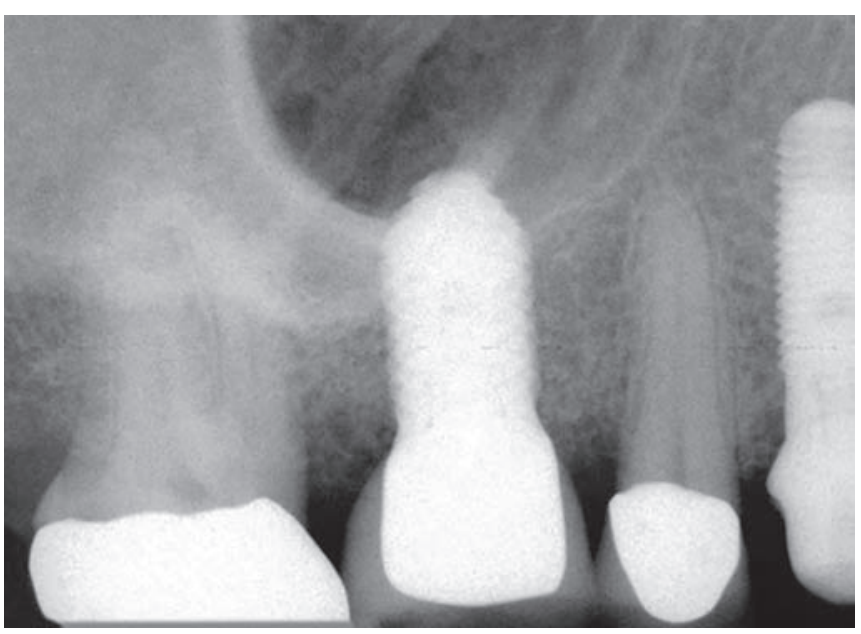

Fig. 19: Final radiograph

lack of soft-tissue closure, flap dehiscence, thin-tissue types, and incongruity between the implant shape and the socket. The OCO Biomedical dental implant used in this case report is designed for immediate loading using Dual Stabilization ${ }^{\mathrm{TM}}$, an industry unique design feature that biomechanically secures the tip and collar of the implant into bone. The Dual Stabilization ${ }^{\mathrm{TM}}$ dental implant achieves Osseous Fixation ${ }^{\mathrm{TM}}$, a biomechanical lock at placement, and is engineered to stimulate bone growth with patented implant features. ${ }^{10}$

\section{CONCLUSION}

Immediate implant placement following tooth extraction has been found to be a viable and predictable solution to tooth loss. This case report illustrates that it is possible to provide the patient with efficient tooth replacement with a quality immediate dental implant. To be successful, implant placement should only be attempted if there is complete resolution of local infection, and if

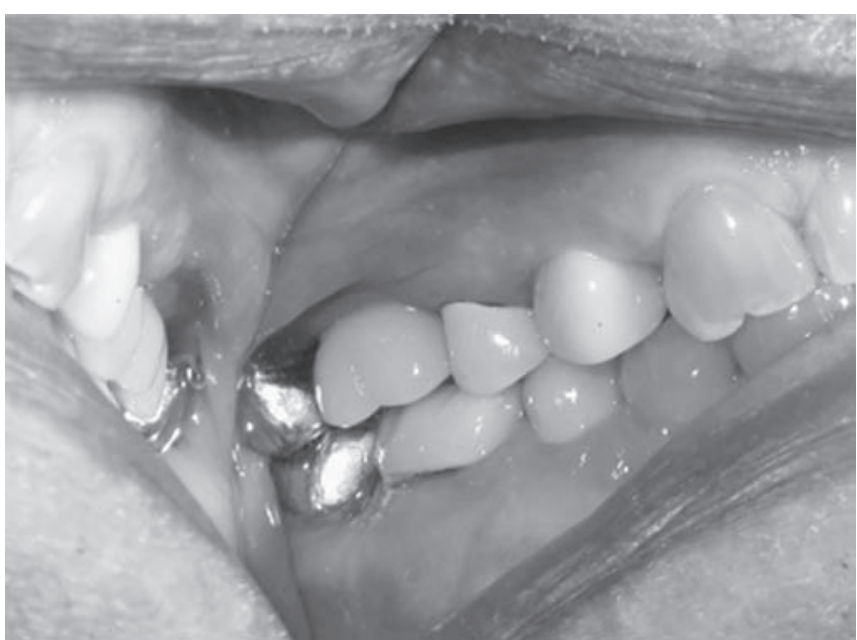

Fig. 18: Final PFG restoration

there is enough bone for placement of an appropriately sized implant, in the ideal restorative position, and with primary implant stability. With changing concepts in technology and incorporation of better implant design, the chances of implant failure can be minimized by using dual stabilization implants that focus on osseous fixation.

\section{REFERENCES}

1. Limor AA, George AZ. Clinical effectiveness of implant supported single tooth replacement. The Toronto study. Int J Oral Maxillofac Implant 1996;11(3):311-321.

2. Branemark PI, Adell R, Breine U, Hansson BO, Lindstrom J, Ohlsson A. Intraosseous anchorage of dental prostheses 1; Experimental studies. Scand J Plast Reconstr Surg 1969;3(2): 81-100.

3. Narayan TV, Sarita JN. Immediate implantation in the anterior maxilla: a case report and discussion of advantages and pitfalls. Int J Oral Implant Clin Res 2010;1(1):33-36.

4. Hämmerle $\mathrm{CH}$, Chen ST, Wilson TG. Consensus statements and recommended clinical procedures regarding the placement of implants in extraction sockets. Int J Oral Maxillofac Implants 2004:19(Suppl):27.

5. Glossary of Oral and Maxillofacial Implants; 2007.

6. Zahnmed SM. Prevention of alveolar ridge resorption after teeth extraction. Int J Oral Maxillofac Surg 2004;114(4):328-336.

7. Esposito M, Grusovin MG, Kwan S, Worthington HV, Coulthard P. Intervention for replacing missing teeth: bone augmentation techniques for dental implant treatment. Aust Dent J 2009;7(4):54.

8. Covani U, Cornelini R, Barone A. Buccolingual bone remodeling around implants placed into immediate extraction sockets: a case series. J Periodontol 2003;74(2):268-273.

9. Novaes A, Vidigal G, Novaes A, Grisi M, Polloni S, Rosa A. Immediate implants placed into infected sites: a histomorphometric study. Int J Oral Maxillofac Impl 1986;13(1):422-427.

10. Saini R. Dental implants: dual stabilization technology. Int J Biomed Adv Res 2015;6(2):196-198. 